\title{
Modélisation de la structure angulaire et du coefficient d'amortissement du champ total d'une source acoustique dans l'océan avec diffusion par la surface agitée et par le fond
}

\author{
M.Yu. GALAKTIONOV
}

IFREMER, Centre de Brest, DITI/SMIASM, BP. 70, 29280 Plouzane, France

\begin{abstract}
One numerical model allowing to study the influence of single and repeted scattering from interfaces of ocean ducts on total field angular structure of one sound source and on field weaking coefficient angular dependence is presented in this paper. The author obtains a system of rayonnement transport equations in functional or differential form as mathematical model for the structure. It is anticipated that the interfaces is omnidirectional and stochastically homogenious. Several exemples of numérical simulation are presented. The scattering on the seas is described with Voronovotch's small-slope approximation or Kurianov's two-scale model. One compares influence of scatterings of different orders.
\end{abstract}

\section{INTRODUCTION}

$\mathrm{La}$ diffusion du son par les interfaces de l'océan influence la structure angulaire du champ d'une source, surtout en assez hautes fréquences. Dans le cas d'une propagation à un grand nombre d'interactions répétées avec des interfaces (propagation "petits fonds", par exemple) des effets de diffusion multiple (DM) aparaissent, et on ne peut plus exclure le champ diffusé du bilan énergétique. Cela mène à une évolution particulière des intervalles de cohérence de signal et donc influence les systèmes de liaison acoustique sous-marine.

Les méthodes de la théorie de transport du rayonnement (TTR) [1] ont été souvent appliquées et ont prouvé leur efficacité pour les problèmes de l'acoustique de l'océan. Nous avons utilisé [2] cette approche pour étudier en simulation l'influence de la DM sur le bruit de l'océan. Dans [3], [4] et dans les plus récents travaux de LYSANOV et BELOUSSOV, cette approche est appliquée pour obtenir un modèle numérique du champ d'une source dans le chenal superficiel à gradient de célérité constant. On retrouve la même équation dans [5], où elle est utilisée pour évaluer les pertes de propagation.

Dans la plupart des cas où on utilise une équation de type de transport du rayonnement comme modèle mathématique du champ acoustique elle peut être obtenue par une voie heuristique et phénoménologique [1]. Dans certains cas [6], elle peut aussi être obtenue à partir de la formulation mathématique qui correspond au problème étudié de propagation, en appliquant les méthodes de la théorie de diffusion multiple.

Dans ce travail, nous nous proposons d'utiliser l'approche de la TTR pour obtenir l'équation qui décrit le champ diffusé aux interfaces dans le guide d'ondes océanique. Nous appliquons ces équations pour modéliser numériquement la structure angulaire du champ d'une source et pour êvaluer l'amortissement du champ diffusé.

\section{PRESENTATION DU MODELE}

Considérons comme modèle de l'océan un guide d'ondes stratifié en immersion $z$, à profondeur constante $H$, avec un profil de célérité dépendant uniquement de l'immersion $z: c=c(z)$. Soit $d \sigma$ une surface élémentaire infiniment petite, $\theta, \varphi$ les deux angles du système de coordonnées sphériques dont l'axe polaire est normal à $d \sigma$. Ces deux angles déterminent le vecteur unité e. Définissons le cofficient de diffusion $m_{s}\left(\mathbf{e}, \mathbf{e}_{0} ; \mathbf{r}\right)$ comme dans [1], à l'aide 
du rapport entre l'énergie $d W(\mathbf{e})$ diffusée dans la direction e et mesurée en un certain point d'observation se trouvant à une distance $R$ de $d \sigma$, et l'énergie $I_{1}\left(e_{o}\right)$ insonofiant la surface $d \sigma$ de la direction $\mathbf{e}_{0}: d W(\mathbf{e})=I_{\mathrm{i}}(\mathbf{e}) \cdot m_{\mathrm{s}}\left(\mathbf{e}, \mathbf{e}_{\mathbf{0}} ; \mathbf{r}\right) \cdot d \sigma / R^{2}$. Par définition, l'intensité de rayonnement du champ diffusé est $I(\mathbf{e})^{\mathrm{d}} \stackrel{\hat{e} \mathrm{f}}{=} d W(\mathbf{e}) / d \mathrm{O}(\mathbf{e})$ où $d \mathrm{o}(\mathbf{e})=d \sigma \cdot R^{-2} \cos \theta$ est l'angle solide sous lequel on voit $d \sigma$ du point d'observation. Par conséquent, $I(\mathbf{e})=I_{\mathrm{i}}\left(\mathbf{e}_{\circ}\right) m_{s}\left(\mathbf{e}, \mathbf{e}_{\mathrm{o}} ; \mathbf{r}\right) / \cos \theta$ et $m$ s doit satisfaire à une condition qui s'écoule de la loi de conservation de l'énergie: $\left(1-\left|V\left(\theta_{0}\right)\right|^{2}\right) \cos \theta_{\circ}=\oint m_{s}\left(\mathbf{e}, \mathbf{e}_{o} ; \mathbf{r}\right) d o(\mathbf{e})$ où $V(\theta)$ est le coefficient de réflexion sur $d \sigma$.

Soit $(0,0, z \circ)$ les coordonnées cartésiennes de l'émetteur, $(\mathbf{x} 1, z 1) \quad\left(\mathbf{x}=\left(x_{1}, 0\right)\right)$ celles du récepteur, les moyennes des ensembles stochastiques des interfaces sont les plans $z=0$ (dit surface) et $z=H$ (dit fond). Introduisons deux indices $\mathrm{p}$ et $\mathrm{d}$ : $\mathrm{p}=\mathrm{s}^{\prime \prime}$ désigne la surface, $\mathrm{p}=" \mathrm{~b}$ " désigne le fond; $\mathrm{d}=$ "+" si $\theta \leq \pi / 2 ; \mathrm{d}=$ "-" si $\pi / 2 \leq \theta \leq \pi$. Notons:

- $z(\theta, z)$ point tournant d'un rayon possédant l'angle $\theta$ à l'immersion $z$;

- $V_{\mathrm{p}}(\theta), m_{s}^{\mathrm{p}}(\ldots)$ coefficients de réflexion et de diffusion par l'interface "p";

- $\mathbb{R}_{\mathrm{p}}, \mathbb{D}_{\mathrm{p}}$ opérateurs de réflexion et de diffusion par l'interface "p":

$\mathbb{R}_{\mathrm{p}}[f](\mathbf{e})=\left|V_{\mathrm{p}}(\mathbf{e})\right|^{2} f(\mathbf{e}), \mathbb{D}_{\mathrm{p}}[f](\mathbf{e})=\oint f\left(\mathbf{e}^{\prime}\right) m_{\mathrm{s}}^{\mathrm{p}}\left(\mathbf{e}, \mathbf{e}^{\prime}\right) / \cos \theta d o\left(\mathbf{e}^{\prime}\right)(\forall f=f(\mathbf{e})) ;$

- $L_{\mathrm{p}}(\theta), X_{\mathrm{p}}(\theta)$ longueur et distance cyclique d'un rayon possédant l'angle $\theta$ près de l'interface "p"; $\mathbf{X}_{\mathrm{p}}(\theta ; \mathbf{e})=X_{\mathrm{p}}(\theta)(\cos \varphi, \sin \varphi, 0)$

- $S_{p}(\theta, \mathbf{e})$ opérateur de déplacement à un demi-cycle: $\varsigma_{p}(\theta, \mathbf{e})[f]=f\left(\mathbf{x}-\mathbf{X}_{\mathrm{p}}(\theta ; \mathbf{e}) / 2\right)(\forall f=f(\mathbf{x}))$;

- $W_{p}(\theta)=\exp \left\{-\beta L_{p}(\theta) / 2\right\}$ facteur d'amortissement dans l'eau sur un demi-cycle; $W_{p}(\theta)$ l'opérateur: $\mathbb{W}_{\mathrm{p}}(\theta)[f](\mathbf{e})=W_{\mathrm{p}}(\theta) f(\mathbf{e})$,

- Ic $(\mathbf{e} ; \mathbf{x}, z)$ intensité du champ cohérent; peut être présentée sous l'approximation géométrique comme la somme des fonctions $A \mathrm{~m}(\mathbf{x}, z) \delta\left(\mathrm{e}-\mathrm{e}_{\mathrm{m}}\right)$ où $A \mathrm{~m}$ sont les carrés d'amplitudes des rayons propres liant le point $(\mathbf{x}, z)$ à l'émetteur et $\mathbf{e}_{\mathrm{m}}$ est la direction du rayon $d^{\prime}$ indice $m$ au point $(\mathbf{x}, z)$.

En suivant [1], [2], nous obtenons pour l'intensité $I(\mathbf{e}, \mathbf{r})$ du champ diffusé le système d'équations de transport:

$$
\begin{aligned}
& I_{+}(\mathbf{e} ; \mathbf{x}, 0)=\mathbb{D}_{s}\left[I_{c_{-}}\left(\mathbf{e}^{\prime} ; \mathbf{x}, 0\right)\right]+\left(\mathbb{R}_{s}+\mathbb{D}_{s}\right)\left[I_{-}\left(\mathbf{e}^{\prime} ; \mathbf{x}, 0\right)\right], \\
& I_{-}(\mathbf{e} ; \mathbf{x}, 0)=(c(z<) / c(0))^{2} W_{s}(\theta) S_{s}(\theta, \mathbf{e})\left[I_{-}(\mathbf{e}<; \mathbf{x}, z<)\right], \quad z<=\min \{z t(\theta, 0), H\}, \\
& I_{-}(\mathbf{e} ; \mathbf{x}, H)=\mathbb{D b}_{b}\left[I_{\mathrm{c}+}\left(\mathbf{e}^{\prime} ; \mathbf{x}, H\right)\right]+(\mathbb{R b}+\mathbb{D b})\left[I_{+}\left(\mathbf{e}^{\prime} ; \mathbf{x}, H\right)\right] \text {, } \\
& I_{+}(\mathbf{e} ; \mathbf{x}, H)=(c(z>) / c(H))^{2} \cdot \mathbb{W b}_{b}(\theta) \operatorname{Sb}(\theta, \mathbf{e})\left[I_{+}(\mathbf{e}>; \mathbf{x}, z>)\right], \quad z>=\max \{z \mathfrak{t}(\theta, H), 0\},
\end{aligned}
$$

où e, e et es sont liées par la loi de Snell-Descartes: $\sin \theta / c(0)=\sin \theta / c(z<)$, $\sin \theta / c(H)=\sin \theta>/ c(z>)$. Dans ce système, $I_{-}$et $I_{+}$aux immersions $0<z<><H$ sont liées aux fonctions $I_{+}(\mathbf{e} ; \mathbf{x}, 0)$ et $I_{-}(\mathbf{e} ; \mathbf{x}, H)$ :

$$
\begin{aligned}
& I_{-}(\mathbf{e}<; \mathbf{x}, z<)=(c(0) / c(z<))^{2} \cdot \mathbb{w}_{s}(\theta) \mathrm{S}_{\mathrm{s}}(\boldsymbol{\theta}, \mathbf{e})\left[I_{+}(\mathbf{e} ; \mathbf{x}, 0)\right] \quad(z<\neq 0, H), \\
& I_{+}(\mathbf{e}>\mathbf{x}, z>)=(c(H) / c(z>))^{2} \cdot w_{b}(\theta) \mathrm{Sb}_{(}(\theta, \mathbf{e})\left[I_{-}(\mathbf{e} ; \mathbf{x}, H)\right] \quad(z>\neq 0, H) .
\end{aligned}
$$

Ce système doit être accompagné de la condition: $\lim \{I(\mathbf{e} ; \mathbf{x}, z)\}=O(|\mathbf{x}|+\infty)$, qui assure le principe d'amortissement aux limites infinies et garanti donc l'absence d'énergie provenant de l'extérieur.

En introduisant les vecteur-colones đc pour l'intensité du champ cohérent et i pour le champ diffusé, nous pouvons écrire le système d'équations de transport sous forme matricielle:

$$
\mathbb{1}=\mathbb{M S} \cdot \mathbb{\|}+\mathbb{D} \cdot \mathbb{d}
$$

Les éléments des matrices $M, S$ et $\mathbb{D}$ ne sont pas présentés, mais $M$ et $\mathbb{D}$ sont les combinaisons des opérateur $S R_{p}, \mathbb{D}_{p}$, et $\$$, a comme éléments les $S_{p}$. La solution formelle de (6) est:

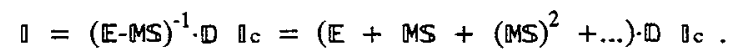

Si on garde dans (5) seulement les termes d'ordre un par rapport à Dp, on aboutit à une solution qui tient compte de la diffusion simple. A partir de cette solution, l'équation correspondante se reconstitue:

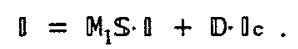

Présentons les équations de transport pour l'intensité de rayonnement dans le cas, où nous avons un seul interface diffusant, disons " $\mathrm{s}$ ":

$$
I+(\mathbf{e} ; \mathbf{x})=\left(\mathbb{R}_{s}+\mathbb{D}_{s}\right) \operatorname{Rb}_{w b}^{2} S_{s}^{2}\left[I_{+}\right]+\mathbb{D}_{s}\left[I_{\mathrm{c}_{-}}\right], \quad I_{+}(\mathbf{e} ; \mathbf{x})=\operatorname{RsRb}_{s} \mathbb{R}^{2} S_{s}^{2}\left[I_{+}\right]+\mathbb{D}_{s}\left[I_{\mathrm{c}_{-}}\right] .
$$

L'éq.(7.2) se différencie de (7.1) par l'absence de l'opérateur $\mathbb{D}_{s} R b W_{b}^{2} S_{s}^{2}$ et elle tient compte uniquement de la diffusion simple. 
Pour simplifier le problème et faciliter les développements théoriques et modélisations numériques, considérons les cas où les interfaces sont omnidirectionnels et homogènes en horizontale. Alors, le coefficient $m_{s}\left(\mathbf{e}, \mathbf{e}_{\mathrm{o}} ; \mathbf{r}\right)$ ne dépend que des angles $\theta, \theta^{\prime}$ et de la différence des angles $\varphi$ et $\varphi_{0}: m_{s}=m_{s}\left(\theta, \theta_{0}, \varphi-\varphi_{0}\right)$. Si l'émission est aussi omnidirectionnelle, il vient de la symétrie du problème que l'intensité $\square(\mathbf{x})$ doit dépendre uniquement de $x=|\mathbf{x}|$. Si nous admettons, de plus, que le lobe du coefficient de diffusion en horizontale est étroit, comme c'est le cas normalement en acoustique sous-marine, nous pouvons l'approcher à l'aide de $m_{s}\left(\mathbf{e}, \mathbf{e}^{\prime}\right) \approx \bar{m}_{s}^{\mathrm{p}}\left(\theta, \theta^{\prime}\right) \delta\left(\varphi-\varphi^{\prime}\right)$, avec $\bar{m}_{s}^{\mathrm{p}}\left(\theta, \theta^{\prime}\right)=\pi_{-\pi}^{\pi} \int m_{s}^{\mathrm{p}}\left(\theta, \theta^{\prime}, \varphi\right) d \varphi / 2 \pi$. Alors, nous cherchons la solution des éq.(4) et (6) sous la forme $\mathbb{1}(x)=\mathbb{\square}(x) \delta(\varphi)$, en estimant que $\overline{\mathrm{\sigma}}(\mathrm{x})={ }_{-\pi}^{\pi} \int \mathrm{\sigma}(\varphi ; \mathrm{x}) d \varphi / 2 \pi$, nous aboutissons toujours aux éq.(4) ou (6) pour $\overline{\mathrm{a}}$, mais cette fois-ci avec l'opérateur $\mathbb{D}_{\mathrm{p}}[f](\theta)=\int_{0}^{\pi / 2} f\left(\theta^{\prime}\right) \overline{m_{s}} \mathrm{p}\left(\theta, \theta^{\prime}\right)\left(\sin \theta^{\prime} / \cos \theta\right) d \theta^{\prime}$. Nous notons ces équations (47), (6). Par conséquent, le problème aux limites de type éliptique posé initialement pour l'équation te transport (4) ou (6) devient un problème aux valeurs initiales de type parabolique (car nous nous débarrassons des ondes rétrodiffusées), et sa solution peut être trouvée numériquement assez facilement.

Admettons pour une approximation que les opérateurs décrivant la diffusion et la réflexion dans l'éq. $\left(4^{\prime}\right)$ sont commutatifs avec l'opérateur de déplacement. Cela exige en fait que $X(\theta) \approx$ const. Cherchons une solution de $\left(4^{\prime}\right)$ là où $I c \ll I$, sous la forme:

$$
\mathbb{1}(\theta ; x)=\Sigma_{\mathrm{k}} e^{-\beta_{\mathrm{k}} x} \cdot \mathrm{v}_{\mathrm{k}}(\theta) \text {, }
$$

où $\left\{V_{k}(\theta), \lambda_{k}\right\}$ sont les vecteur-colones et les valeurs propres de l'opérateur M. Alors, nous retrouvons: $\beta_{\mathrm{k}}=-2 \ln \left\{\lambda_{\mathrm{k}}\right\} X$. Par conséquent, les valeurs $\operatorname{Re}\left\{\beta_{\mathrm{k}}\right\}$ ainsi calculées joue le rôle des coefficients effectifs d'amortissement exponentiel du champ diffusé dans le guide d'ondes. Nous pouvons les comparer avec les coefficients d'amortissement du champ cohérent: $\beta_{c}=\left[\beta L_{s}(\theta)-\ln \left\{\left|V_{s}(\theta) V_{b}(\theta b(\theta))\right|^{2}\right\}\right] / X_{s}(\theta)$.

$\mathrm{Si}$ l'on veut négliger la structure d'interférence de large échelle et considérer la moyenne $\tilde{a}(x)$ sur la plus grande distance cyclique $X_{\mathrm{m}}$, alors, là où $x \gg X_{\mathrm{m}}$, nous pouvons approcher l'opérateur $\$$ à l'aide des deux premiers termes de la série de Teylor pour $\tilde{0}$. De ce lissage de l'éq.(4') vient l'équation de transport de forme intégro-differentielle, utilisée dans [3], [4], par exemple. Dans ce cas, nous pouvons retrouver de la même manière les coefficients $\beta k$. Au fait, pour pouvoir appliquer cette deuxième méthode de calcul du coefficient d'amortissement, on doit supposer que l'amortissement sur un cycle est faible. Cette supposition permet d'établir le lien entre les deux méthodes d'évaluation de l'amortissement. Notons aussi que les coefficients d'amortissement ainsi calculés tiennent compte de la redistribution de l'énergie acoustique due à la DM par les interfaces du guide d'ondes océanique, ainsi que d'autres pertes d'énergie telles que l'absorbtion dans l'eau et dans le fond.

\section{SIMULATION NUMERIQUE}

Pour retrouver les solutions des éq.(4) et $\left(6^{\prime}\right)$, nous avons élaboré une procédure numérique de calcul des fonctions $I_{+}(\theta ; x, 0)$ et $I_{-}(\theta ; x, H)$. Alors, pour obtenir le spectre angulaire du champ diffusé à l'immersion de réception $z 1$ avec une résolution donnée en angle, il faut faire partir de ce point tous les rayons selon ce pas angulaire et les poursuivre jusqu'aux interfaces. Alors, les valeurs des fonctions $I_{ \pm}$pour les angles liés aux angles de départ selon la loi de Snell, corrigées à l'aide des facteurs $\left(c_{\mathrm{p}} / c_{(z 1}\right)^{2}$ et de ceux d'amortissement correspondant à ces arcs des rayons, donnent les valeurs recherchées $I(\theta ; x 1, z 1)$. Afin de faire des estimations pour l'océan, nous utilisons l'approximation des inclinaisons faibles [7], ainsi que les approches traditionnelles: des perturbations faibles, de Kirchoff et à deux échelles.

Comme résultats des simulations numériques, nous présentons sur les figures 1 et 2 quelques exemples de spectres angulaires du champ diffusé selon le système (1)-(2), ceci pour le vent $8 \mathrm{~m} / \mathrm{s}$ et deux fréquences ( 1 et $3 \mathrm{kHz}$ ) dans les conditions de propagation "grands fonds", avec un chenal profond (type "G") et un chenal superficiel (type "J"). Les courbes inférieures présentent la solution de l'éq. $\left(6^{\prime}\right)$ (diffusion simple), et les barres verticales présentent les amplitudes des rayons propres.

Une procédure numérique pour retrouver les fonctions propres et les coefficients $\beta_{k}$ est également élaborée. Les simulations montrent (fig.3a) que presque toute fonction propre possède un maximum aigu pour un certain angle $\theta_{\mathrm{k}}$. Par conséquent, chaque valeur $\beta_{\mathrm{k}}$ détermine essentiellement l'amortissement de l'énergie du champ en distance pour les angles au 
voisinage de celui de maximum. On peut donc établir une dépendance approchée de $\beta(\theta)$ présentée sur les figures $3 \mathrm{~b}, \mathrm{c}$ avec $\beta_{\mathrm{c}}(\theta)$.

\section{CONCLUSION}

Dans un chenal sous-marin aux interfaces perturbées, le champ diffusé d'une source acoustique a un caractère zonal déterminé par les zones de convergence.

La diffusion multiple accroît le champ total et peut mener à l'apparition de faux rayons (fig.1a). Cet effet crôt avec la vitesse du vent et avec la fréquence, et il est maximal au voisinage de $2 \mathrm{kHz}$. En même temps, la diffusion multiple aide la diffusion simple à lisser le spectre angulaire du champ total. Par exemple, à la fréquence $3 \mathrm{kHz}$, cela est déjà sensible à $50 \mathrm{~km}$ de la source, et à $100 \mathrm{~km}$ on ne peut plus sélectionner énergétiquement les familles de rayons dans le champ (fig.2).

Les deux méthodes de calcul du coefficient d'amortissement du champ diffusé aboutissent à des résultats proches pour les angles $\theta$ rasants (supérieurs à $72^{\circ}$ ). Aux angles inférieurs à $70^{\circ}$, l'approche de l'équation différentielle aboutit à des valeurs surestimées de $\beta(\theta)$ et $\beta_{c}(\theta)$. Comme il fallait s'y attendre, le coefficient d'amortissement du champ cohérent est supérieur à celui du champ diffusé. Dans un chenal superficiel la différence de ces deux coefficients est plus grande aux angles rasants tandis que dans un chenal profond elle est plus grande pour des angles inférieurs à $70^{\circ}$. Cet effet s'explique par le fait que les rayons rasants subissent le plus de contacts avec l'interface diffusant si l'axe du chenal se trouve sur lui, et ils en subissent le moins si ce n'est pas le cas. Nous avons donc démontré que les effets de diffusion interviennent différemment selon les conditions de propagation.

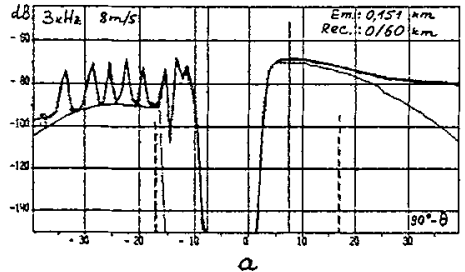

$a$

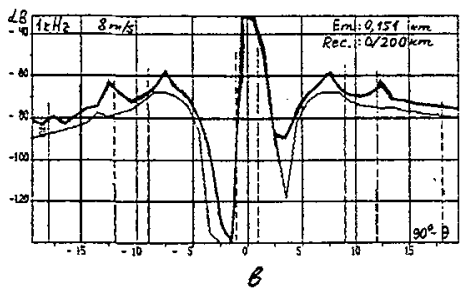

Fig.1. Spectres angulaires du champ diffusé Chenal profond type " $G$ ".
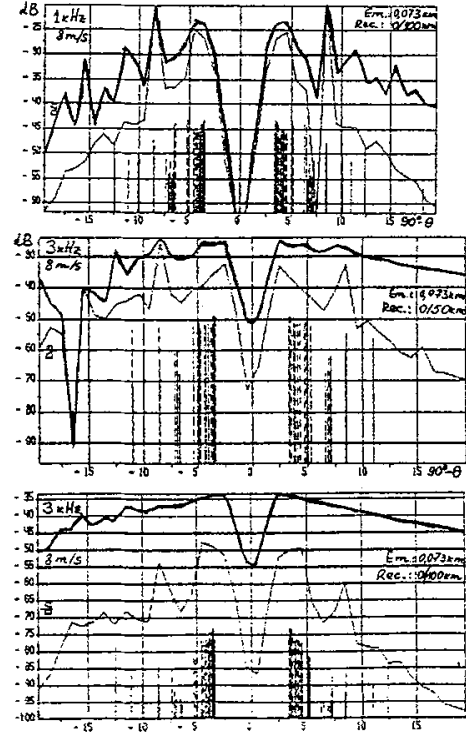

Fig.2. Spectres angulaires du champ diffusé

Chenal superficiel type "J".
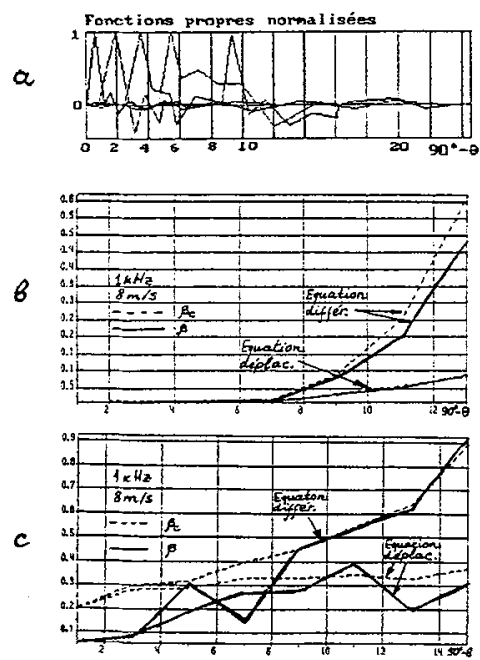

Fig.3. Amortissement du champ diffusé: a-fonctions propres; b-chenal "G"; c-chenal "J".

\section{REFERENCES}

[1] Ishimaru A., Wave Propagation and Scattering in Random Media (Academic Press, New York, San Francisco, London, 1978).

[2] Galaktionov M., "Calcul de la structure angulaire du bruit sous-marin avec réflexions multiples sur la houle", 2ème CFA, Arcachon France avril 1992, J.Roux Ed., (Les Editions de Physique, Les Ulis, 1992) v.II, pp.949-952.

[3] Wilson H.L., Tappert F.D., J.A.S.A., 66(1979), pp.256-274.

[4] Beloussov A.V., Lysanov I.P., Sudostroenie, série "Acoustique", 1986, n.1, pp.73-79.

[5] Lurton X., "Application de la modélisation géométrique de la propagation acoustique sous-marine en petits fonds et hautes fréquences", 12ème Colloque GRETSI, 1989.

[6] Borodin V.V., J.d'Acoustique, Acad. des Sc. d'URSS, 33(1987), n.4, pp.588-598.

[7] Galaktionov M., J.d'Acoustique, Acad. des Sc. d'URSS, 37(1991), n.2, pp.270-276. 